\title{
Mercurio total en bagre rayado y bocachico del río Meta, Colombia
}

\section{Total mercury in striped catfish and bocachico from river Meta, Colombia}

\author{
Santiago Valbuena-Rodríguez ${ }^{1}$; Miguel Ángel Navarro-Ramírez ${ }^{2}$
}

\begin{abstract}
${ }^{1}$ Químico, M.Sc. Universidad de los Llanos, Grupo de investigación Quimera. Villavicencio - Meta, Colombia; e-mail: svalbuena@ unillanos.edu.co; iD https://orcid.org/0000-0002-9034-6640

2Químico, Ph.D. Universidad de los Llanos, Grupo de investigación Quimera. Villavicencio - Meta, Colombia; e-mail: miguel.navarro@ unillanos.edu.co; iD https://orcid.org/0000-0002-5535-4784
\end{abstract}

Cómo citar: Valbuena-Rodríguez, S.; Navarro-Ramírez, M.Á. 2021. Mercurio total en bagre rayado y bocachico del río Meta, Colombia. Rev. U.D.C.A Act. \& Div. Cient. 24(2):e1880. http://doi.org/10.31910/rudca.v24.n2.2021.1880

Artículo de acceso abierto publicado por Revista U.D.C.A Actualidad \& Divulgación Científica, bajo una Licencia Creative Commons CC BY-NC 4.0

Publicación oficial de la Universidad de Ciencias Aplicadas y Ambientales U.D.C.A, Institución de Educación Superior Acreditada de Alta Calidad por el Ministerio de Educación Nacional.

Recibido: febrero 27 de 2020

Aceptado: agosto 30 de 2021

Editado por: Ingeborg Zenner de Polanía

\section{RESUMEN}

El consumo de pescado contaminado con mercurio representa un grave problema para la salud humana, en especial, en poblaciones, en las cuales, forma parte de su dieta diaria. Este problema requiere un seguimiento de la concentración de mercurio en peces depredadores y no depredadores de agua dulce, especialmente, en zonas poco estudiadas, como la región de la Orinoquia colombiana. Por lo tanto, el objetivo de este estudio fue determinar la concentración de mercurio total en muestras de bagre rayado (Pseudoplatystoma fasciatum metaense) y de bocachico (Prochilodus mariae), de la parte alta del río Meta, Colombia, peces muy consumidos por las poblaciones aledañas. Ocho muestras de cada especie de pez se analizaron por duplicado, por espectrofotometría de absorción atómica, con gene-rador de hidruros. Se estableció, que el método aplicado es trazable al material de referencia certificado Dorm-4; también, se realizó la validación del método. El valor promedio de mercurio total en bagre rayado fue de $0,055 \pm 0,0107 \mathrm{mg} / \mathrm{kg}$ y para el bocachico, 0,026 $\pm 0,0054 \mathrm{mg} / \mathrm{kg}$ de pescado fresco. Las muestras analizadas no sobrepasan el valor límite establecido por la Organización Mundial de la Salud y Environmental Protection Agency; sin em-bargo, el índice de riesgo por efectos no cancerígenos, en algunas muestras de bagre rayado, presentan un valor superior a uno, por lo que su consumo representa un riesgo para la salud, especialmente, durante la gestación y la primera infancia, así como de pescadores y aquellas comunidades de la región, que dependen del consumo de este alimento.

Palabras clave: Contaminación ambiental; Mercurio; Pez; Salud pública.

\section{ABSTRACT}

The consumption of fish contaminated with mercury represents a serious problem for human health, especially in populations in which it is part of their daily diet. This problem requires the moni-toring of mercury concentration in freshwater predatory and non-predatory fish, especially in poorly studied areas such as the Orino-quia region of Colombia. The aim of this study was to determine total mercury concentration in samples of catfish (Pseudoplatystoma fasciatum metaense) and bocachico (Prochilodus mariae) 
from the river Meta, Meta-Colombia. Fishes very much consumed by the adjacent populations. Eight samples of each fish species were analyzed in duplicate by atomic absorption spectrophotometry with a hydride generator. It was established that the applied method is traceable to the Dorm-4 certified reference material; the method was also validated. The average value of total mercury in striped catfish was $0.055 \pm 0.0107 \mathrm{mg} /$ $\mathrm{kg}$, and for bocachico $0.026 \pm 0.0054 \mathrm{mg} / \mathrm{kg}$ of fresh fish. These data obtained do not exceed the limit value established by the World Health Organization and Environmental Protection Agency. However, the risk index for non-carcinogenic effects in some samples of striped catfish have a value greater than one, therefore their consumption represents a health risk, especially during pregnancy and early childhood, as well as for fishermen and those communities in the area that depend on the consumption of this food.

Keywords: Environmental pollution; Fish; Mercury; Public health.

\section{INTRODUCCIÓN}

El mercurio es un metal tóxico que genera graves problemas en la salud pública y ambiental (Guzzi et al. 2021; OMS, 2017). El efecto tóxico, se explica mediante su ciclo biogeoquímico, el cual, muestra que este metal no se elimina de la naturaleza, sino que se reconvierte de sus formas inorgánicas a las orgánicas.

El mercurio metálico $\mathrm{Hg}^{0}$, por sus propiedades físicas únicas, tiene una alta presión de vapor, por lo que es volatilizado a la atmósfera (González-Raymat et al. 2017) y es fácilmente transportado en forma de gas, a muchas partes del planeta. Se puede oxidar lentamente $\mathrm{a} \mathrm{Hg}^{2+}$, por efecto del ozono o precipitarse en la superficie de la tierra, como deposición húmeda, por las aguas lluvias; absorberse por las raíces de las plantas; depositarse en la nieve o en las fuentes de aguas naturales, en los sedimentos, en el suelo o por deposición en seco, en donde una parte se reconvierte en $\mathrm{Hg}^{0}$, que vuelve a la atmósfera (Morel et al. 1998; Branfireun et al. 2020).

Algunos microorganismos, a través de una reacción de metilación, convierten el mercurio en metilmercurio (MeHg) (Bravo \& Cosio, 2020), siendo acumulado en el fitoplancton y zooplancton. Estos últimos representan el alimento de peces iliófagos o piscívoros, generando su bioacumulación y, posteriormente, a través de la cadena alimentaria, cuando se pasa de un nivel trófico inferior a otro superior, al aumentar su concentración en diferentes especies de peces depredadores, mediante el proceso de biomagnificación (Pecoraro et al. 2019; Zhang et al. 2020).

El metilmercurio es tóxico para la salud humana, presentando actividad neurotóxica, citoxicidad, genotoxidad, toxicidad cardiovascular, cambios hormonales y del metabolismo en mamíferos (Farina \& Aschner, 2019; Yang et al. 2020), siendo de mayor relevancia su efecto, en los niños durante su gestación y su primera infancia (Ha et al. 2017; Santos-Lima et al. 2020).
El mercurio de origen antropogénico genera una problemática de contaminación ambiental grave, cuyas emisiones mundiales a la atmosfera, para el 2015, fueron calculadas en 2.200 t. El sector más influyente en el total de esta emisión fue la minería de oro artesanal a pequeña escala (Artisanal and Small Scale Gold Mining, ASGM), con $37,7 \%$, seguido de la quema de combustibles fósiles y biomasa, con $24 \%$; la producción de metal no ferroso, con $15 \%$; industria del cemento, $11 \%$; los residuos de productos con mercurio, el $7 \%$; la industria de metales ferrosos, $2 \%$ y otras fuentes, el $2 \%$ (UNEnvironment, 2019).

Por su parte, Asia contribuye en esta emisión, con $49 \%$; América del Sur, $18 \%$ y África Subsahariana, con el $16 \%$, estos últimos, principalmente, a través de la minería aurífera artesanal a pequeña escala (emisión de 1.220 t, para el 2015), por lo cual, ha generado el denominado legado de mercurio, la forma más influyente en el histórico de finales del siglo XIX, inclusive, con una emisión antropogénica mayor a todas las industrias del siglo XX (UNEnvironment, 2019).

En América, las consecuencias de ASGM se han evidenciado, inicialmente, en peces, con concentraciones de mercurio mayores a la norma internacional $0,5 \mathrm{mgHg} / \mathrm{kg}$, en pescado fresco (Silva et al. 2019; Silva et al. 2020). En Colombia, específicamente, en la cuenca del río Magdalena y sus afluentes (Mancera-Rodríguez \& ÁlvarezLeón, 2006), en la región de la Mojana (Marrugo-Negrete et al. 2020) y en el caso de la Orinoquía (Cala, 2001; Trujillo et al. 2010).

Otros estudios en Colombia han determinado la concentración de mercurio en habitantes de zonas cercanas a la explotación minera artesanal de oro (Salazar-Camacho et al. 2017; GutiérrezMosquera et al. 2018; Valdelamar-Villegas \& Olivero-Verbel, 2020), demostrando que esta población presenta contaminación por mercurio.

Debido a los pocos estudios respecto a contaminación por mercurio en peces de la región de la Orinoquía colombiana, el objetivo de este trabajo fue determinar las concentraciones de mercurio total en muestras de pescado fresco de bagre rayado y de bocachico, de la parte alta del río Meta, en el sector de Puerto López, dos especies importantes desde el punto de vista comercial y de consumo. Asimismo, evaluar los parámetros para determinar la validez del método y el riesgo que puede representar para la salud humana, el consumo de estas especies de peces.

\section{MATERIALES Y MÉTODOS}

Sitio de estudio y muestreo. Las muestras de pescado, se recolectaron en agosto del 2016, en época de lluvia, por pescadores en la zona, llamada "bocas del Guayuriba", parte alta del río Meta, localizada en la confluencia de los ríos Metica y Guayuriba, de la Orinoquía colombiana, en las coordenadas $356^{\prime} 22.27^{\prime \prime} \mathrm{N}$ y $735^{\prime} 55.41^{\prime \prime O}$ (Figura 1), a una altura de $197 \mathrm{~m}$ s.n.m., con una precipitación promedio de $2.700 \mathrm{~mm}$, una temperatura media de 28 ${ }^{\circ} \mathrm{C}$ y con un $77 \%$ de humedad relativa (Ajiaco et al. 2015). 
El muestreo, se realizó de acuerdo con la guía para la evaluación de contaminantes químicos (Environmental Protection Agency, EPA, 2000); de esta forma, fueron capturados ocho especímenes de bagre rayado (Psendoplatystoma fasciatum-metaense-Familia Pimelodidae) y ocho de bocachico (Prochilodus mariae-Familia Prochilodontidae), caracterizados por su sexo, tamaño en $\mathrm{cm}$ y peso en $\mathrm{kg}$ y luego eviscerados. Posteriormente, se extrajeron porciones de tejido muscular sin piel, que fueron guardadas en bolsas de plástico y conservadas a $-20{ }^{\circ} \mathrm{C}$.
Análisis de mercurio. La determinación de la concentración de mercurio total $\left(\mathrm{Hg}_{\mathrm{T}} \mathrm{T}\right)$, se realizó mediante la técnica de espectrofotometría de absorción atómica con generador de hidruros, siguiendo la metodología descrita por la Association of Official Agricultural Chemists, AOAC (método oficial 977.15 AOAC, 1978). El material de laboratorio para el análisis del mercurio, se lavó previamente, con ácido nítrico al $10 \%(\mathrm{~V} / \mathrm{V})$. La digestión de las muestras, se realizó en presencia de $\mathrm{V}_{2} \mathrm{O}_{5}$, con

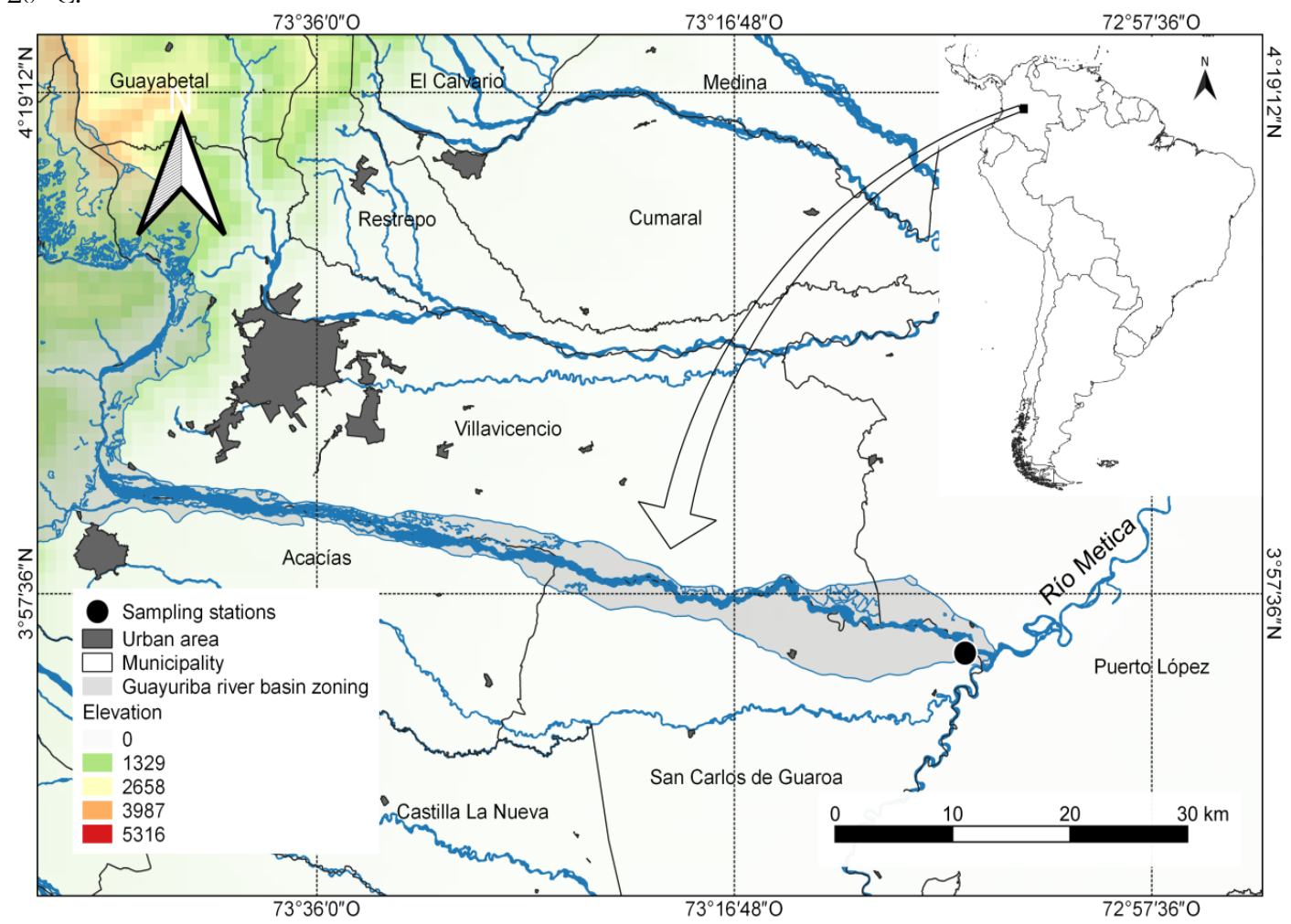

Figura 1. Ubicación de la zona de muestreo. Mapa creado con QGIS.

una mezcla de $\mathrm{H}_{2} \mathrm{SO}_{4}-\mathrm{HNO}_{3}$ (1:1). Se usó agua tipo 1, con una conductividad de $0,05 \mu \mathrm{S} / \mathrm{cm}$. Todos los reactivos usados en el análisis y la extracción del mercurio en el tejido muscular de las muestras de pescado fueron grado analítico.

Evaluación de la calidad del método. La calidad de los resultados del análisis de mercurio total, se evaluó de acuerdo con las normas ISO/IEC-norma 17025 (ISO/IEC, 2005), AOAC y la red de organizaciones en Europa para mediciones químicas (Ellison \& Williams, 2019), validación, incertidumbre y trazabilidad), a partir de la integración de tres elementos claves: la trazabilidad, la validación del método y la incertidumbre de las mediciones.

La trazabilidad del método, se realizó por comparación con el Material de Referencia Certificado (MRC), Dorm-4 del National Research Council Canada, aplicando la prueba t de Student, para conocer si hubo o no diferencia estadísticamente significativa entre la concentración de $\mathrm{Hg}$ T del MRC y la concentración obtenida experimentalmente en un único laboratorio. La repetibilidad y la precisión intermedia, se determinó mediante el diseño anidado, de acuerdo con Magnusson \& Örnemark (2014), con la aplicación de análisis de varianza de un factor ANOVA. La validación del método (Tabla 1), se realizó mediante los criterios de Magnusson \& Örnemark (2014).

La incertidumbre de la concentración de $\mathrm{Hg}$-T en las muestras de pescado, se expresa como la media \pm la incertidumbre expandida (U), en $\mathrm{mg}$ de $\mathrm{Hg} / \mathrm{kg}$, mediante la ecuación 1, como lo reporta el consejo nacional de investigación de Canadá, para el MRC Dorm-4.

$\mathrm{U}=k \mu_{\mathrm{c}}$

ecuación 1

En la que $\mathrm{k}$ es el factor de cobertura, cuyo valor es 2 y $\mu_{\mathrm{c}}$, es la incertidumbre combinada.

La $\mu_{\mathrm{c}}$, se obtiene mediante la ecuación 2:

$\mu_{\mathrm{c}}=2 \sqrt{ } \mu_{\text {proc. }}^{2}+\mu_{\text {traz }}^{2}$ ecuación 2 
Donde, $\mu_{\text {proc }}$ es la incertidumbre del procedimiento analítico obtenida de ANOVA derivada de la varianza de la serie y de la repetibilidad y $\mu_{\text {traz }}$ es la varianza asociada al MRC y a la varianza del método.
Análisis estadístico. Se aplicó ANOVA con estudio de diseño anidado, para conocer la precisión en la trazabilidad y la prueba de Mandel, para la linealidad y la precisión de la validación del método; se realizó la prueba t de Student en la verificación de la

Tabla 1. Parámetros de la validación del método de análisis de mercurio por AAS.

\begin{tabular}{|c|c|c|c|}
\hline \multicolumn{2}{|c|}{ Parámetro } & Determinación & Observaciones \\
\hline \multicolumn{2}{|l|}{ Linealidad } & $\begin{array}{l}\text { Cinco curvas patrón de } \\
\text { concentración de mercurio de } \\
10,20,30,40 \text { y } 50 \mu g \mathrm{Hg} / \mathrm{L}\end{array}$ & $\begin{array}{l}\text { Evaluada, a través del } \\
\text { coeficiente de regresión } \\
\text { lineal y mediante la } \\
\text { prueba de Mandel }\end{array}$ \\
\hline \multicolumn{2}{|c|}{ Límite de detección } & $\begin{array}{l}\text { Diez blancos sin analito y diez } \\
\text { muestras fortificadas con } \\
\text { mercurio } 3,00 \mu \mathrm{g} / \mathrm{L}\end{array}$ & $\begin{array}{l}\mathrm{LD}=\mathrm{yB}+3 \mathrm{x} 0 \\
\begin{array}{llll}\text { Donde, yB es media } & \text { de } \\
\text { respuesta } & \text { instrumental del } \\
\text { blanco y } & \text { s0 es } & \text { la } \\
\text { desviación estándar de } & \text { la } \\
\text { respuesta } & \text { instrumental del } \\
\text { blanco }\end{array}\end{array}$ \\
\hline \multicolumn{2}{|l|}{ Exactitud } & $\begin{array}{l}\text { Porcentaje de recuperación en } \\
\text { tres niveles de concen- } \\
\text { tración }\end{array}$ & $\begin{array}{lr}\text { Porcentajes } & \text { recomendados } \\
\text { por la } & \text { AOAC } \\
\text { para concentraciones } & \text { en trazas } \\
\text { de analito } & \end{array}$ \\
\hline \multirow[b]{2}{*}{ Precisión } & Repetibilidad & 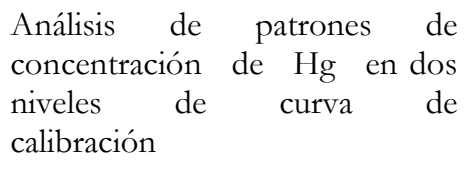 & $\begin{array}{l}\text { Coeficiente de variación, } \\
\text { según el criterio de } \\
\text { AOAC para trazas analito }\end{array}$ \\
\hline & $\begin{array}{l}\text { Precisión } \\
\text { intermedia }\end{array}$ & $\begin{array}{l}\text { Mediciones de } \mathrm{Hg} \text { a una } \\
\text { concentración intermedia } \\
\text { de } 28 \mu \mathrm{g} / \mathrm{L}, \quad \text { por dos } \\
\text { analistas diferentes en días } \\
\text { diferentes }\end{array}$ & $\begin{array}{l}\text { ANOVA, para evaluar si } \\
\text { hubo } \\
\text { significativas } \\
\text { mediciones } \\
\text { entre los analistas, en los días } \\
\text { diferentes }\end{array}$ \\
\hline
\end{tabular}

trazabilidad. Se aplicó la prueba de Mann-Whitney, para conocer si había diferencias en los niveles de $\mathrm{Hg}$ entre las especies y entre sexo; para el caso de la misma especie, se usó el software R.

Índice de riesgo (Hazard Index, HI). Se calculó de acuerdo con U.S.EPA (1989), mediante la ecuación 3:

$\mathrm{HI}=[\mathrm{MeHg}] * \mathrm{I} / \mathrm{RfD} . \mathrm{W}$ ecuación 3
Donde, $[\mathrm{MeHg}]$ es la concentración de metilmercurio en $\mu \mathrm{g} / \mathrm{g}$ obtenida, considerando que el $95 \%$ del Hg-T encontrado en peces adultos es meHg (Bloom, 1992; Lescord et al. 2018); I, una ingesta diaria de $100 \mathrm{~g}$ (UNEP/WHO, 2008); W es el peso corporal en $\mathrm{kg}$ (en la mujer promedio de $60 \mathrm{~kg}$ y para el hombre de $70 \mathrm{~kg}$ ) y $\mathrm{RfD}$ es la dosis de referencia para el $\mathrm{MeHg}$, con un valor de 0,1 $\mu \mathrm{g} / \mathrm{kg} /$ día (U.S.EPA, 1989). Un valor de HI mayor a uno $(1,0)$, sugiere un alto riesgo para la salud humana. 
Tabla 2. Concentraciones de $\mathrm{Hg} \mathrm{T}(\mathrm{mg} / \mathrm{Kg})$ y $\mathrm{HI}$ de las muestras de bagre rayado y bocachico.

\begin{tabular}{|c|c|c|c|c|c|c|c|c|}
\hline \multicolumn{9}{|c|}{ Bocachico } \\
\hline (n) & 1 & 2 & 3 & 4 & 5 & 6 & 7 & 8 \\
\hline $\begin{array}{l}\text { Peso } \\
(\mathrm{kg})\end{array}$ & 0,98 & 1,05 & 0,96 & 1,10 & 0,99 & 1,00 & 0,97 & 0,99 \\
\hline $\begin{array}{l}\text { Tamaño } \\
(\mathrm{cm})\end{array}$ & 37 & 39 & 36 & 41 & 37 & 38 & 36 & 37 \\
\hline Sexo & $\mathrm{m}$ & $\mathrm{h}$ & $\mathrm{m}$ & $\mathrm{H}$ & $\mathrm{m}$ & $\mathrm{h}$ & $\mathrm{m}$ & $\mathrm{m}$ \\
\hline $\begin{array}{l}\mathrm{HgT}] \\
\dot{\mathrm{X}} \pm \mu\end{array}$ & $\begin{array}{c}0,021 \pm \\
0,004\end{array}$ & $\begin{array}{c}0,034 \pm \\
0,007\end{array}$ & $\begin{array}{c}0,022 \pm \\
0,005\end{array}$ & $\begin{array}{c}0,033 \pm \\
0,007\end{array}$ & $\begin{array}{c}0,023 \pm \\
0,005\end{array}$ & $\begin{array}{c}0,030 \pm \\
0,006\end{array}$ & $\begin{array}{c}0,024 \pm \\
0,005\end{array}$ & $\begin{array}{c}0,020 \pm \\
0,004\end{array}$ \\
\hline $\begin{array}{l}\text { HI } \\
\text { Mujer } \\
(60 \mathrm{~kg})\end{array}$ & 0,33 & 0,54 & 0,35 & 0,52 & 0,37 & 0,48 & 0,38 & 0,32 \\
\hline $\begin{array}{l}\text { HI } \\
\text { Hombre } \\
(70 \mathrm{~kg})\end{array}$ & 0,29 & 0,46 & 0,30 & 0,44 & 0,31 & 0,41 & 0,33 & 0,27 \\
\hline \multicolumn{5}{|c|}{ Promedio $=0,026 \pm 0,005 \mathrm{mg} / \mathrm{kg}$} & \multicolumn{4}{|c|}{ Rango 0,020-0,034mg/kg } \\
\hline \multicolumn{9}{|c|}{ Bagre rayado } \\
\hline (n) & 1 & 2 & 3 & 4 & 5 & 6 & 7 & 8 \\
\hline $\begin{array}{l}\text { Peso } \\
(\mathrm{kg})\end{array}$ & 4,5 & 8,0 & 8,5 & 5,1 & 4,9 & 5,8 & 4,7 & 4,8 \\
\hline $\begin{array}{l}\text { Tamaño } \\
(\mathrm{cm})\end{array}$ & 78 & 97 & 108 & 82 & 93 & 94 & 81 & 85 \\
\hline Sexo & $\mathrm{m}$ & $\mathrm{h}$ & $\mathrm{h}$ & $\mathrm{m}$ & $\mathrm{m}$ & $\mathrm{h}$ & $\mathrm{m}$ & $\mathrm{m}$ \\
\hline $\begin{array}{l}\mathrm{HgT}] \\
\dot{\mathrm{X}} \pm \mu\end{array}$ & $\begin{array}{c}0,021 \pm \\
0,004\end{array}$ & $\begin{array}{c}0,077 \pm \\
0,014\end{array}$ & $\begin{array}{c}0,176 \pm \\
0,033\end{array}$ & $\begin{array}{c}0,034 \pm \\
0,007\end{array}$ & $\begin{array}{c}0,032 \pm \\
0,007\end{array}$ & $\begin{array}{c}0,050 \pm \\
0,009\end{array}$ & $\begin{array}{c}0,022 \pm \\
0,005\end{array}$ & $\begin{array}{c}0,029 \pm \\
0,006\end{array}$ \\
\hline $\begin{array}{l}\text { HI } \\
\text { Mujer } \\
(60 \mathrm{~kg})\end{array}$ & 0,33 & 1,22 & 2,79 & 0,53 & 0,50 & 0,80 & 0,35 & 0,47 \\
\hline $\begin{array}{l}\text { HI } \\
\text { Hombre } \\
(70 \mathrm{~kg})\end{array}$ & 0,29 & 1,04 & 2,39 & 0,46 & 0,43 & 0,69 & 0,30 & 0,40 \\
\hline \multicolumn{5}{|c|}{ Promedio $=0,055 \pm 0,0107 \mathrm{mg} / \mathrm{kg}$} & \multicolumn{4}{|c|}{ Rango 0,021-0,176mg/kg } \\
\hline
\end{tabular}

$\dot{\mathrm{X}} \pm \mu=$ promedio de $\mathrm{Hg} \mathrm{T}(\mathrm{mg} / \mathrm{kg}) \pm$ incertidumbre expandida

\section{RESULTADOS Y DISCUSIÓN}

Las muestras de pescado analizadas no sobrepasaron los valores establecidos por la norma nacional e internacional de $0,5 \mathrm{mgHg} / \mathrm{kg}$ pescado fresco (UNEP/WHO, 2008; Minsalud, 2012), pero todas presentaron contaminación con mercurio (Tabla 2).

Los estudios realizados en la misma zona del río Meta, por Cala (2001) y Trujillo et al. (2010), en bagre rayado, muestran también valores por debajo de la norma; en estos estudios, no se incluyó el análisis de HgT de peces, como el bocachico, el cual, es importante, ya que es una especie detritívora, ubicada en el nivel más bajo de la cadena alimentaria que, al consumir fitoplancton y zooplancton del sedimento o materia orgánica en descomposición contaminada, bioacumula el mercurio en forma de metilmercurio (Pecoraro et al. 2019). Este mercurio es absorbido y acumulado en el tejido muscular y, luego, se concentra en los peces depredadores, pasando al hombre, a través del consumo de pescado contaminado, promoviendo la bioacumuación y biomagnificación. 
Los valores de HI para las muestras de bocachico, tanto para la mujer en edad fértil como adultos, no representan ningún riesgo en la salud, para un consumo de pescado diario de $100 \mathrm{~g}$, mientras que, en el caso de la especie de bagre rayado, en algunas muestras, se determinó un HI mayor a uno, lo cual, genera una voz de alerta respecto al riesgo para el cerebro en formación, cuyo efecto adverso principal del metilmercurio es la neurotoxidad, que se presenta con síntomas, como parálisis cerebral, pérdida de visión y audición, trastornos en el desarrollo (Lee et al. 2020) y afecta críticamente las funciones neuropsicológicas (Clarkson, 2002; Santos-Lima et al. 2020).

Este riesgo es eminente, si se tiene en cuenta que los ribereños de la región dependen de este alimento para su subsistencia y es seguro que el consumo de pescado por estas personas es mayor a $100 \mathrm{~g} /$ día.

Un consumo mayor a $100 \mathrm{~g}$ de pescado diario o mayor a $700 \mathrm{~g}$ por semana, excedería la ingesta máxima provisional (ISTP, en adultos es de $3,2 \mu \mathrm{g} / \mathrm{kg}$ de peso corporal/semana), calculada por organismos internacionales, como la Food Agriculture Organization (FAO/OMS, 2017), por lo que se recomienda no exceder este consumo, especialmente, en mujeres embarazadas y niños $(1,6 \mu \mathrm{g} / \mathrm{kg}$ de peso corporal/semana).

En esta región, la presencia de pescado contaminado con mercurio, se puede explicar, a través de la contaminación por ASGM, ubicada en la cuenca del río Inírida, afluente del río Orinoco. En el proceso de extracción del oro, una parte del mercurio es vaporizado a la atmósfera y, otra parte, es transferida a las fuentes naturales de aguas. La contaminación directa ha sido evidenciada a partir de los niveles de mercurio en sangre y cabello de los pescadores del Guainía (Ldrovo et al. 2001).

Por su parte, el mercurio liberado al medio ambiente puede ser transportado y convertido según el ciclo del mercurio, de las formas inorgánicas a las orgánicas y luego transformado en metilmercurio, en los sedimentos. Estos sedimientos son la fuente de alimento de peces, como el bocachico que, a su vez, es consumido por el bagre. El bagre, especie de gran capacidad de migración transfronteriza, se puede desplazar desde el río Orinoco hacia la parte alta del río Meta (Zapata \& Usma, 2013), explicando, de esta forma, la bioacumulación y biomagnificación del mercurio, en peces de la región.

La calidad de los resultados, se soportó en la trazabilidad, la validación (Tabla 3) y la incertidumbre del método. La trazabilidad, se determinó, a través de la prueba t de Student, con tcalc $<$ ttabla para $\mathrm{gl}=8, \mathrm{p}<0,05$ no se encontraron diferencias significativas entre la concentración de Hg-T certificada del MRC (0,412 \pm 0,036 $\mathrm{mg} / \mathrm{kg}$ ) y la concentración media obtenida experimentalmente en el labo-ratorio $(0,414 \pm 0,072 \mathrm{mg} / \mathrm{kg})$, mientras la recuperación analítica del MRC, se encontró en el rango esperado de 99,50 a $101,41 \%$, permitiendo concluir que, el método aplicado por el laboratorio, es trazable al material de referencia.

Tabla 3. Validación del método de análisis de mercurio por AAS.

\begin{tabular}{|c|c|c|}
\hline \multicolumn{2}{|c|}{ Parámetro } & Resultado \\
\hline \multicolumn{2}{|l|}{ Linealidad } & $\begin{array}{l}\text { Ecuación de la curva y= 0,015X - 0,0108 } \\
\text { Coeficiente de correlación lineal R2=0,999 } \\
\text { Test Mandel- ANOVA- p 0,897>0,05 }\end{array}$ \\
\hline \multicolumn{2}{|c|}{ Límite de detección } & $0,015 \mathrm{mgHg} / \mathrm{kg}$ \\
\hline \multicolumn{2}{|l|}{ Exactitud } & $\begin{array}{l}\text { Porcentaje de recuperación entre } 98 \text { y 107\% } \\
\text { Coeficiente variación CV menor a 5,18\% }\end{array}$ \\
\hline \multirow{2}{*}{ Precisión } & Repetibilidad & $\begin{array}{l}\text { Patrón de baja concentración con desviación } \\
\text { estándar de } 0,365 \mu \mathrm{gHg} / \mathrm{L} \text { y un CV de } 2,42 \% \\
\text { Patrón de alta concentración con desviación } \\
\text { estándar de } 0,629 \mu \mathrm{gHg} / \mathrm{L} \text { y un CV fue de } 1,41 \%\end{array}$ \\
\hline & $\begin{array}{l}\text { Precisión } \\
\text { intermedia }\end{array}$ & $\begin{array}{l}\text { Como Fcalc }<\text { Ftabla, gl }=7, \mathrm{p}<0,05 \text {, se concluye que } \\
\text { no existen diferencias significativas en los ensayos } \\
\text { realizados en el laboratorio }\end{array}$ \\
\hline
\end{tabular}


La concentración de $\mathrm{HgT}$ para las muestras de pescado, se calcularon por relación proporcional al valor de la trazabilidad del método obtenido en el MRC Dorm-4, expresada como el promedio de la muestra \pm incertidumbre expandida, $\dot{X} \pm \mu$.

La incertidumbre del procedimiento analítico, en el caso de las muestras de pescado, se corrigió por presentar una incertidumbre estándar relativa mayor en la precisión intermedia del método para una concentración baja $(15 \mu \mathrm{gHg} / \mathrm{L})$, con un valor de $0,0242 \mu \mathrm{gHg} / \mathrm{L}$, mientras que la incertidumbre estándar relativa de la concentración alta $(45 \mu \mathrm{gHg} / \mathrm{L})$, presentó un valor de $0,0141 \mu \mathrm{gHg} / \mathrm{L}$. Esto condujo a obtener dos valores diferentes de incertidumbre expandida, una incertidumbre para la concentración baja de analito, calculada en $0,087 \mathrm{mgHg} / \mathrm{kg}$, mientras que la incertidumbre para la concentración alta de analito fue de $0,077 \mathrm{mgHg} / \mathrm{kg}$.

Los resultados de la prueba de Mann-Whitney ( $\mathrm{p}>0,05)$ indicaron que no existen diferencias significativas en la concentración de $\mathrm{Hg}$ en las dos especies; sin embargo, se encontraron diferencias significativas $(\mathrm{p}<0,05)$ en el sexo, con una concentración de $\mathrm{Hg}$ mayor en las hembras de bagre rayado, las cuales, presentaron mayor tamaño (Tabla 2).

De acuerdo con los hallazgos de esta investigación, todas las muestras analizadas de bocachico y de bagre rayado, presentaron contaminación con mercurio, con un valor mínimo de $0,020 \mathrm{mg} / \mathrm{kg}$ y máximo de $0,176 \mathrm{mg} / \mathrm{kg}$; destacando las hembras de mayor masa de la especie bagre, como las de mayor concentración, evidenciando una mayor concentración en peces de mayor nivel trófico; sin embargo, estos valores no sobrepasan el valor máximo aceptable $(0,5 \mathrm{mgHg} / \mathrm{kg})$. Es tan importante determinar el nivel de mercurio en peces como el índice de riesgo, ya que, en este caso, el cálculo del HI, determinó valores mayores a uno (1), indicando un riesgo para su consumo, por lo que se debe controlar y limitar el consumo de bagre rayado de los ribereños del río Meta, a una ingesta semanal inferior a $700 \mathrm{~g}$, en busca de evitar problemas de salud, generados por efectos neurotóxicos del metilmercurio, especialmente, en la mujer embarazada, para proteger el feto en formación, sin afectar, en mayor medida, su estilo de vida.

Se recomienda realizar estudios de la concentración de mercurio en aguas, suelos y sedimentos en esta región, así como el análisis en el cabello y sangre de los habitantes de las riberas del río Meta. Estos estudios permitirán continuar con el monitoreo de la contaminación con este metal y evaluar su impacto en la salud, lo cual, facilitará, posteriormente, la formulación e implementación de planes de contingencia y de mejoramiento de las condiciones de vida de estas comunidades.

Agradecimientos. A la Universidad de los Llanos, por el apoyo económico en la ejecución del proyecto de investigación "Estudio preliminar de las concentraciones de mercurio total en en tejido muscular de Psendoplatystoma fasciatum y de Procbilodus mariae del río Meta, zona de Puerto López, departamento del Meta”, de código C03-F02-009-2015. Conflicto de intereses: El manuscrito fue elaborado y revisado con la participación de todos los autores, quienes declaran que no existe ningún conflicto de intereses que pueda cuestionar la validez de los resultados presentados.

\section{REFERENCIAS}

1. AJIACO, R.E.; RAMÍREZ, H.; BOLAÑOS, J.A. 2015. La pesquería en Bocas del Guayuriba, alto del río Meta, Orinoquia Colombiana. Editorial Universidad de los Llanos (Colombia). 89p.

2. ASSOCIATION OF OFFICIAL AGRICULTURAL CHEMISTS, AOAC. 1978. Official Method 977.15 Mercury in Fish. Alternative Flameless Atomic Absorption Spectrophotometric Method, First Action 1977, Final Action 1978. (United States).

3. BLOOM, N.S. 1992. On the chemical form of mercury in edible fish and marine invertebrate tissue. Canadian J. Fisheries and Aquatic Sciences. 49(5):1010-1017. https://doi.org/10.1139/f92-113

4. BRANFIREUN, B.A.; COSIO, C.; POULAIN, A.J.; RIISE, G.; BRAVO, A.G. 2020. Mercury cycling in freshwater systems - An updated conceptual model. Sci. Total Environ. (Netherlands). 745:140906. https://doi.org/10.1016/j.scitotenv.2020.140906

5. BRAVO, A.G.; COSIO, C. 2020. Biotic formation of methylmercury: A bio-physico-chemical conundrum. Limnol. Oceanogr. (United States). 65(5):1010-1027. https://doi.org/10.1002/lno.11366

6. CALA, P. 2001. Occurrence of mercury in some commercial fish species from the Magdalena and Meta rivers in Colombia. Rev. Asociación Colombiana de Ictiología. 4:15-19.

7. CLARKSON, T.W. 2002. The three modern faces of mercury. Environ. Health Perspect. (United States). 110(Supp.1):11-23. https://doi.org/10.1289/ehp.02110s111

8. ELLISON, S.L.R.; WILLIAMS, A. 2019. Eurachem/CITAC Guide: Metrological Traceability in Analytical measurement. Disponible desde Internet en: www.eurachem.org (con acceso el 06/04/2020).

9. ENVIRONMENTAL PROTECTION AGENCY, EPA. 2000. Guidance for Assessing Chemical Contaminant Data for Use in Fish Advisories. Volume 1. Fish Sampling and Analysis. Third Edition. (United States). Disponible desde Internet en: 
https://www.epa.gov/sites/production/files/2018-11/ documents/guidance-assess-chemical-contaminantvol1-third-edition.pdf (con acceso en 03/02/2020).

10. FARINA, M.; ASCHNER, M. 2019. Glutathione antioxidant system and methylmercury-induced neurotoxicity: An intriguing interplay. Biochim. Biophys Acta Gen. Subj. (Netherlands). 1863(12):129285.

https://doi.org/10.1016/j.bbagen.2019.01.007

11. FOOD AND AGRICULTURE ORGANIZATION/ORGANIZACIÓN MUNDIAL DE LA SALUD (FAO/OMS) 2017. Comisión del Codex Alimentarius. Programa Conjunto FAO/OMS Sobre Normas Alimentarias. Comité del Codex Sobre Contaminantes de los Alimentos. 11 Reunión, Rio de Janeiro, Brasil 3-7 de abril de 2007.

12. GONZÁLEZ-RAYMAT, H.; LIU, G.; LIRIANO, C.; LI, Y.; YIN, Y.; SHI, J.; JIANG, G.; CAI, Y. 2017. Elemental mercury: Its unique properties affect its behavior and fate in the environment. Environmental Pollution (United Kingdom). 229:69-86.

http://dx.doi.org/10.1016/j.envpol.2017.04.101

13. GUTIÉRREZ-MOSQUERA, H.; SUJITHA, S.B.; JONATHAN, M.P.; SARKAR, S.K.; MEDINAMOSQUERA, F.; AYALA-MOSQUERA, H.; MORALES-MIRA， G.; ARRE-OLA-MENDOZA， L. 2018. Mercury levels in human population from a mining district in Western Colombia. J. Envi-ron. Sci. (China). 68:83-90.

https://doi.org/10.1016/j.jes.2017.12.007

14. GUZZI, G.; RONCHI, A.; PIGATTO, P. 2021. Toxic effects of mercury in humans and mammals. Chemosphere (United Kingdom). 263:127990.

https://doi.org/10.1016/j.chemosphere.2020.127990

15. HA, E.; BASU, N.; BOSE-O’REILLY, S.; DÓREA, J.G.; MCSORLEY, E.; SAKAMOTO, M.; CHAN, H.M. 2017. Current progress on understanding the impact of mercury on human health. Environ. Res (United States). 152:419-433.

https://doi.org/10.1016/j.envres.2016.06.042

16. ISO/IEC. 2005. ISO/IEC-17025. General requirements for the competence of testing and calibration laboratories. ISO/IEC. Geneva, Suiza.

17. LDROVO, A.J.; MANOTAS, L.E.; VILLAMIL, G.; ORTIZ, J.; SILVA, E.; ROMERO, S.A.; AZCARATE, E. 2001. Niveles de mercurio y percepción de riesgo en una población aurífera del Guainía (Orinoquia colombiana). Biomédica. 21(2):134-41.

https://doi.org/10.7705/biomedica.v21i2.1101

18. LEE, J.-Y.; HWANG, G.-W.; NAGANUMA, A.; SATOH, M. 2020. Methylmercury toxic mechanism related to protein degradation and chemokine transcription. Environ. Health
Prev. Med (Japan). 25(1):30.

https://doi.org/10.1186/s12199-020-00868-3

19. LESCORD, G.; JOHNSTON, T.; BRANFIREUN, B.; GUNN, J. 2018. Percentage of methylmercury in the muscle tissue of freshwater fish varies with body size and age and among species. Environ. Toxicol. Chem (United States). 37(10):2682-2691. https://doi.org/10.1002/etc.4233

20. MAGNUSSON, B.; ÖRNEMARK, U. 2014. Eurachem Guide: The Fitness for Purpose of Analytical Methods - A Laboratory Guide to Method Validation and Related Topics. Disponible desde Internet en: www.eurachem.org (con acceso el 24/07/2020).

21. MANCERA-RODRÍGUEZ, N.J.; ÁLVAREZ-LEÓN, R. 2006. Estado del conocimiento de las concentraciones de mercurio y otros metales pesados en peces dulceacuícolas de Colombia. Acta Biol. Colomb. 11(1):3-23.

22. MARRUGO-NEGRETE, J.; VARGAS-LICONA, S.; RUIZGUZMÁN, J.A.; MARRUGO-MADRID, S.; BRAVO, A.; DÍEZ, S. 2020. Human health risk of methylmercury from fish consumption at the largest floodplain in Colombia. Environ. Res. (United States) 182:109050. https://doi.org/10.1016/j.envres.2019.109050

23. MINISTERIO DE SALUD Y PROTECCIÓN SOCIAL. 2012. Minsalud Resolución 122 de 2012. Ministerio de salud y protección social. Disponible desde Internet en: https://www.minsalud.gov.co/sites/rid/Lists/ BibliotecaDigital/RIDE/DE/DIJ/Resolucion-122de-2012.pdf (con acceso el 14/08/2021).

24. MOREL, F.; KRAEPIEL, A.; AMYOT, M. 1998. The Chemical cycle and bioaccumulation of mercury. Annu. Rev. Ecol. Evol. Syst. (United States). 29:543-566.

https://doi.org/10.1146/annurev.ecolsys.29.1.543

25. OMS. 2017. El mercurio y la salud. Organización Mundial de la Salud Noticias. Disponible desde Internet en:

https://www.who.int/es/news-room/fact-sheets/detail/ mercury-and-health (con acceso el 21/08/2021).

26. PECORARO, G.D.; HORTELLANI, M.A.; HAGIWARA, Y. S.; BRAGA, E.S.; SARKIS, J.E.; AZEVEDO, J.S. 2019. Bioaccumulation of total mercury (THg) in catfish (Siluriformes, Ariidae) with different sexual maturity from Cananéia-Iguape Estuary, SP, Brazil. Bull. Environ. Contam. Toxicol. (United States). 102(2):175-179. https://doi.org/10.1007/s00128-018-2485-3

27. SALAZAR-CAMACHO, C.; SALAS-MORENO, M.; MARRUGO-MADRID, S.; MARRUGO-NEGRETE, J.; DÍEZ, S. 2017. Dietary human exposure to mercury in two artisanal small-scale gold mining communities of 
northwestern Colombia. Environ. Int. (United Kingdom) 107:47-54.

https://doi.org/10.1016/j.envint.2017.06.011

28. SANTOS-LIMA, C.D.; MOURÃO, D.D.S.; CARVALHO, C.F.D.; SOUZA-MARQUES, B.; VEGA, C.M.; GONÇALVES, R.A.; ARGOLLO, N.; MENEZES-FILHO, J.A.; ABREU, N.; HACON, S.D.S. 2020. Neuropsychological effects of mercury exposure in children and adolescents of the Amazon Region, Brazil. Neurotoxicology (Netherlands). 79:48-57.

https://doi.org/10.1016/j.neuro.2020.04.004

29. SILVA, L.; ABREU, I.; FERREIRA DA C.N., A.; RODRIGUES, B.W.; MAGALHÃES, M.C. 2020. Mercury concentration in six fish guilds from a floodplain lake in western Amazonia: Interaction between seasonality and feeding hab-its. Ecol. Indic. (Netherlands). 111:106056.

https://doi.org/10.1016/j.ecolind.2019.106056

30. SILVA, S.F. DA.; OLIVEIRA, D.C.; PEREIRA, J.P.G.; CASTRO, S.P.; COSTA, B.N.S.; LIMA, M. DE O. 2019. Seasonal variation of mercury in commercial fishes of the Amazon Triple Frontier, Western Amazon Basin. Ecol. Indic. 106:105549.

https://doi.org/10.1016/j.ecolind.2019.105549

31. TRUJILLO, G.F.; LASSO, C.A.; DIAZGRANADOS, M.C.; FARINA, O.; PÉREZ L.E.; BARBARINO, A.; GONZÁLEZ, M. 2010. Evaluación de las concentraciones de mercurio en peces de interés comercial en ecosistemas acuáticos de la Orinoquia. Biodiversidad de la cuenca del Orinoco. Bogotá: Unión Gráfica. 355p.

32. UN-ENVIRONMENT. 2019. Global Mercury Assessment 2018. UN Environment Programme, Chemicals and Health Branch Geneva, Switzerland. Disponible desde Internet en: https://www.unep.org/resources/publication/globalmercury-assessment-2018 (con acceso el 13/10/2020).
33. UNEP/WHO. 2008. Guidance for identifying populations at risk from mercury exposure. United Nations environment programme/world health organization.

34. U.S.EPA UNITED STATES ENVIRONMENTAL PROTECTION AGENCY USEPA. 1989. Risk Assessment Guidance for Superfund, vol. 1, Human Health Evaluation Manual, EPA/540/1-89/002. U.S. Environmental Protec-tion Agency. (United States). Disponible desde Internet en: https://www.epa.gov/ sites/production/files/2015-09/documents/rags_a.pdf (con acceso el 14/05/2019).

35. VALDELAMAR-VILLEGAS, J.; OLIVERO-VERBEL, J. 2020. High Mercury levels in the indigenous population of the Yaigojé Apaporis National Natural Park, Colombian Amazon. Biol. Trace Elem. Res. (United States). 194(1):3-12. https://doi.org/10.1007/s12011-019-01760-0

36. YANG, L.; ZHANG, Y.; WANG, F.; LUO, Z.; GUO, S.; STRAHLE, U. 2020. Toxicity of mercury: molecular evidence. Chemosphere (United Kingdom). 245:125586. https://doi.org/10.1016/j.chemosphere.2019.125586

37. ZAPATA, L.A.; USMA, J.S. 2013. Guía de las especies Migratorias de la Biodiversidad en Colombia. Peces. Vol. 2. Ministe-rio de Ambiente y Desarrollo Sostenible / WWFColombia. Bogotá, D.C. Colombia. 486p. Disponible desde Internet en: https://wwflac.awsassets.panda.org/ downloads/migratoriaspeces_42_web_final.pdf (con acceso 15/06/2019).

38. ZHANG, Y.; SOERENSEN, A.L.; SCHARTUP, A.T.; SUNDERLAND, E.M. 2020. A global model for methylmer-cury formation and uptake at the base of marine food webs. Global Biogeochemical Cycles (United States). 34(2):e2019GB006348.

https://doi.org/10.1029/2019GB006348 